\title{
AS FINALIDADES DO ORÇAMENTO NA INFLUÊNCIA DO ISOMORFISMO INSTITUCIONAL NA AUTORREJEIÇÃO
}

\author{
The budget purposes on the influence of institutional isomorphism on \\ self-rejection
}

Igor Pereira da Luz

E-mail: i.pereiradaluz@gmail.com

Mestre em Contabilidade pelo Programa de Pós-graduação em Contabilidade da Universidade

Federal de Santa Catarina; Doutorando no Programa de Pós-graduação em Contabilidade da Universidade Federal de Santa Catarina; Professor no Curso de Ciências Contábeis da Faculdade Estácio de Sá de São José, SC e da Faculdade Energia. Endereço para contato: Avenida Leoberto Leal, 431, Barreiros, 88117-001, São José, Santa Catarina, Brasil. https://orcid.org/0000-0002-3932-3611

Carlos Eduardo Facín Lavarda E-mail: elavarda@gmail.com

Doutor em Contabilidade pela Universidade de Valência, Espanha, homologado pela Universidade de São Paulo; Mestre em Ciências Contábeis pela Universidade do Vale do Rio dos Sinos; Professor na Graduação e na Pós-graduação do Departamento de Ciências Contábeis da Universidade

Federal de Santa Catarina. https://orcid.org/0000-0003-1498-7881 
O presente trabalho teve por objetivo examinar a influência do isomorfismo institucional na autorrejeição ao orçamento, mediada pelas finalidades do artefato. Aponta-se a importância do estudo do setor farmacêutico brasileiro pois este representa um exemplo peculiar de discussão institucional, considerando as pressões de isomorfismo institucional sofridas pelo setor. $O$ estudo é descritivo, de abordagem quantitativa, caracterizado como pesquisa de levantamento, tipo survey. Para cumprir o objetivo foram utilizados os pressupostos de DiMaggio e Powell (1983), as respostas estratégicas de desafiar e manipular do modelo conceitual de Oliver (1991) e as finalidades do orçamento utilizadas por Ekholm e Wallin (2011). A população selecionada foram os gerentes das empresas do setor farmacêutico vinculadas ao Guia da Farmácia, e a amostra final foi composta por 147 respostas válidas. Para a análise de dados foi utilizada a Modelagem de Equações Estruturais. Os resultados apontam para uma influência positiva do isomorfismo institucional nas finalidades de planejamento e diálogo. Não foi encontrada, porém, influência das finalidades do orçamento na autorrejeição. $O$ isomorfismo institucional ainda apresentou influência positiva na autorrejeição do orçamento. Como contribuições, o estudo traz indícios de que os fatores de isomorfismo levam a uma melhor utilização das finalidades do orçamento, levam também a uma rejeição do artefato, que pode estar ligada a fatores de multiplicidade e restrição.

Palavras-chave: Finalidades do orçamento. Respostas estratégicas. Teoria Institucional. Isomorfismo institucional.

\section{Abstract}

The study aimed to examine the influence of institutional isomorphism on self-rejection to the budget, mediated by the artifact's purposes. It is important to study the Brazilian pharmaceutical sector, as it represents a peculiar example of institutional discussion, considering the pressures of institutional isomorphism suffered by the sector. The study is descriptive, with a quantitative approach, characterized as survey research. to achieve the research objective, the assumptions of DiMaggio and Powell (1983), the strategic responses to challenge and manipulate Oliver's conceptual model (1991) and the budget purposes used by Ekholm and Wallin (2011) were used. The selected population was the managers of companies in the pharmaceutical sector linked to the Guia da Farmácia, and the final sample consisted of 147 valid responses. For data analysis, the Structural Equation Modeling was used. The study results show a positive influence of institutional isomorphism in the purposes of planning and dialogue. However, the influence of the budget's purposes on self-rejection was not found. Institutional isomorphism still had a positive influence on budget self-rejection. As contributions, the study brings evidence that the factors of isomorphism lead to a better use of the purposes of the budget, also lead to a rejection of the artifact, which may be linked to factors of multiplicity and restriction.

Keywords: Budget purposes. Strategic responses. Institutional Theory. Institutional isomorphism.

\section{INTRODUÇÃO}

Utilizados como meio para facilitar a condução dos processos políticos entre os indivíduos e orientados por valores que englobam uma pluralidade de interesses dentro da complexidade na qual estão inseridos, os orçamentos são um dos tópicos mais pesquisados na literatura de artefatos de gestão (Covaleski, Evans, Luft, \& Shields, 2003). Existe ainda 
uma preocupação com o artefato no que se refere à sua prática (Hansen, Otley, \& Van der Stede, 2003), visto que sua implementação e manutenção no ambiente organizacional leva em conta uma série de recursos da organização (Frezatti, Aguiar, \& Rezende, 2007).

Atrelada a esses fatores, parte da literatura critica a utilização do orçamento nas organizações (Libby \& Lindsay, 2010). Entre as principais críticas, os estudos apontam que o artefato gera apenas mudanças incrementais, sendo uma barreira para mudanças, verticalizando os processos e encorajando jogos de interesses entre os envolvidos (Neely, Sutcliff, \& Heyns, 2001), o que torna o mecanismo pouco eficiente, distanciando as organizações de suas metas. No mais, tem-se que os orçamentos não são sensíveis aos ambientes que mudam rapidamente (Hansen et al., 2003).

Apesar dessas frequentes críticas, os orçamentos continuam sendo amplamente utilizados nas organizações (Kenno, Lau \& Sainty, 2018; Libby \& Lindsay, 2010) e a literatura aponta alguns avanços visando alternativas para os problemas apresentados, como utilizar orçamentos contínuos atualizando com mais frequência os planos, substituindo os padrões fixos anuais por metas flexíveis (Hansen et al., 2003; Kenno et al., 2018). No mais, os orçamentos não são estáticos e podem assumir diversas finalidades no contexto organizacional (Hansen \& Van der Stede, 2004).

Essas finalidades podem levar a diferentes respostas dos envolvidos no processo (Hansen \& Van der Stede, 2004). Com isso, faz-se importante considerar que o orçamento não opera de forma isolada (Hansen et al., 2003), sendo influenciado por pressões econômicas, sociais e organizacionais do ambiente que englobam as práticas organizacionais e influenciam as organizações e suas práticas (Scapens, 2006).

Essas pressões fazem com que as organizações sejam forçadas a mudanças nas suas lógicas institucionais, tornando-as mais homogêneas, em um processo nomeado de isomorfismo institucional (DiMaggio \& Powell, 1983; Zucker, 1987). Essas mudanças ocorrem na busca em atender a um conjunto de expectativas visando obter legitimidade frente ao ambiente em que estão inseridas, e estão ligadas à aceitação de procedimentos socialmente institucionalizados (Meyer \& Rowan, 1977).

Nesse escopo, Oliver (1991) alerta que, além de considerar os efeitos do ambiente entre a conformidade estrutural e o isomorfismo institucional, é importante considerar o papel da resistência nas relações entre organização e ambiente, havendo uma ampla gama de respostas estratégicas para o ambiente institucional que irão variar de acordo com as pressões institucionais exercidas sobre as organizações (Oliver, 1991).

Oliver (1991) ainda aponta que fatores preditivos podem levar a uma maior rejeição da institucionalização dos artefatos gerencias, como o orçamento. Por exemplo, o orçamento como artefato para atender a uma multiplicidade de interesses ou como fator de restrição 
das decisões dos envolvidos são situações que podem levar a uma maior autorrejeição ao orçamento nas organizações.

No mais, as organizações têm diferentes razões potenciais para a utilização do orçamento, e a pouca utilização dessas finalidades pode levar a uma maior rejeição do artefato (Hansen \& Van der Stede, 2004), visto que uma maior utilização das finalidades tem minimizado percepções negativas sobre o orçamento no contexto organizacional (Hansen \& Van der Stede, 2004; Mucci, Frezatti, \& Dieng, 2016).

Nesse contexto surge a seguinte pergunta de pesquisa: qual a influência do isomorfismo institucional na autorrejeição, mediada pelas finalidades do orçamento? A partir do problema proposto, foi elaborado o objetivo do estudo de: examinar a influência do isomorfismo institucional na resposta estratégica de rejeição ao orçamento, mediada pelas finalidades do artefato.

O presente trabalho contribui para a literatura orçamentária embasada pela Teoria Institucional, pois traz à discussão a autorrejeição frente às pressões institucionais, que tem sido pouco explorada no escopo do artefato (Wijethilake, Munir, \& Appuhami, 2017). O estudo justifica-se ainda quanto à sua originalidade, visto que trouxe à discussão a proposta para a utilidade das finalidades do artefato na relação isomorfismo institucional e autorrejeição dos envolvidos.

Justifica-se a escolha pelo setor farmacêutico brasileiro pois este representa um exemplo interessante da discussão institucional, sofrendo de regulamentações governamentais, como controle de preços, pressões sociais e políticas exercidas a partir da regulação econômica (PricewaterhouseCoopers, 2018; Sindusfarma, 2018). No mais, o setor apresenta expressivos números de crescimento (PricewaterhouseCoopers, 2018; Sindusfarma, 2018), fazendo com que essas organizações tenham uma incerteza maior no ambiente organizacional, processo que leva ao isomorfismo mimético (DiMaggio \& Powell, 1983). E acarretando em maior profissionalização, levando ao isomorfismo normativo (DiMaggio \& Powell, 1983).

\section{FUNDAMENTAÇÃO TEÓRICA}

\subsection{ISOMORFISMO INSTITUCIONAL E O SETOR FARMACÊUTICO BRASILEIRO}

Mudanças organizacionais fazem parte do ciclo de vida das organizações, que visam sobreviver ao ambiente em que estão inseridas. Essas mudanças são provenientes de pressões institucionais exercidas pelo meio no qual as organizações se encontram (Guerreiro, Pereira, Rezende, \& Aguiar, 2010). Muitas dessas mudanças vêm em busca da legitimidade. As 
organizações buscam essa legitimação pelas facilidades que ela poderia trazer na obtenção de recursos e sobrevivência em seus ambientes, assegurando, assim, acesso aos recursos essenciais (Meyer \& Rowan, 1977).

DiMaggio e Powell (1983) afirmam que em resposta às pressões institucionais exercidas sobre as organizações, estas tendem a adotar práticas e estruturas de organizações que estão em contexto similares. Esse processo de pressões institucionais leva à homogeneização das organizações, nomeado de isomorfismo; ele tem sido utilizado como base teórica em estudos para investigar as questões das mudanças organizacionais (DiMaggio \& Powell, 1983). DiMaggio e Powell elencam três mecanismos que levam a mudanças institucionais: o isomorfismo coercitivo, o isomorfismo mimético e o isomorfismo normativo.

O isomorfismo coercitivo é resultante das pressões formais e informais exercidas sobre as organizações por stakeholders dos quais elas sejam dependentes. Muitas das mudanças coercitivas são respostas diretas a regulamentações governamentais, pressões sociais, culturais e políticas exercidas sobre uma organização (DiMaggio \& Powell, 1983). A literatura aponta que as empresas são mais suscetíveis às pressões coercivas quando dependem de recursos escassos, como capital, matrizes controladoras, clientes e fornecedores (Brandau, Endenich, Trapp, \& Hoffjan, 2013).

No caso do setor farmacêutico, o fator coercitivo ocorre pelas regulamentações governamentais, como controle de preços, pressões sociais e políticas exercidas a partir da regulação econômica, visto a importância dos produtos para a sociedade em geral (PricewaterhouseCoopers, 2018; Sindusfarma, 2018) No contexto brasileiro, essa situação também é impulsionada pelo capital externo inserido na economia nacional e a crescente exportação dos produtos, com isso as organizações têm uma relação de dependência com as matrizes internacionais (PricewaterhouseCoopers, 2018; Sindusfarma, 2018).

Além das pressões, outro fator que leva ao isomorfismo é a incerteza no ambiente. Quando as organizações se defrontam com problemas de causas ambíguas ou com soluções pouco nítidas, uma alternativa é copiar as práticas de outras organizações, o que se constitui como isomorfismo mimético, impulsionado por tecnologias defasadas e objetivos ambíguos (DiMaggio \& Powell, 1983). No setor farmacêutico a incerteza maior no ambiente organizacional é ocasionada pelos expressivos números de crescimento (PricewaterhouseCoopers, 2018; Sindusfarma, 2018), fazendo com que essas organizações tenham que se adaptar às práticas estabelecidas pelo setor (DiMaggio \& Powell, 1983).

Tem-se ainda a mudança decorrente da profissionalização dos agentes das organizações, como fator de isomorfismo, nomeado de isomorfismo normativo. Essa profissionalização é definida como a busca coletiva dos membros para definir as condições e os métodos de seu trabalho e estabelecer uma base cognitiva e legítima para a sua autonomia ocupacional. 
Como as mesmas categorias profissionais sofrem as mesmas pressões coercitivas e miméticas, estas tendem a utilizar as mesmas estratégias (DiMaggio \& Powell, 1983).

Achados da literatura apontam que as práticas de contabilidade gerencial são utilizadas como respostas às pressões institucionais, e que os mecanismos isomórficos afetam a institucionalização ou não dessas práticas nas organizações (Amans, Mazars-Chapelon, \& Villesèque-Dubus, 2015; Cohen \& Karatzimas, 2014).

Soma-se a isso a escassez de evidências apresentadas pelo contexto farmacêutico (Gomes, Pimentel, Cardoso, \& Pieroni, 2014). Assim, o setor farmacêutico brasileiro representa um exemplo interessante, a partir de suas peculiaridades e da discussão institucional, e pode auxiliar no entendimento de organizações que potencialmente enfrentam fortes pressões.

\subsection{RESPOSTA DE AUTORREJEIÇÃO}

Apesar da contribuição da literatura institucional, baseada no isomorfismo institucional, sobre organizações e ambiente em que estão inseridas, os estudos nessa vertente tendem a ignorar o papel da agência ativa dos envolvidos no processo e os graus de resistência que podem ocorrer nas relações entre organização e ambiente (Oliver, 1991).

Visando a essa variação nas dimensões do comportamento organizacional, Oliver (1991) estabelece as bases conceituais para a identificação de estratégias alternativas, em resposta ao ambiente institucional. A autora argumenta que os agentes envolvidos no processo de institucionalização nem sempre possuem um comportamento passivo frente às instituições, e suas respostas dependem dos fatores preditivos das pressões institucionais.

Essas respostas às pressões institucionais, dependendo dos fatores preditivos, podem ser mais ativas indicando um menor grau ou até a não institucionalização dos arranjos estruturais (Frezatti et al., 2007). Nesse conceito, Oliver (1991) elabora as respostas estratégicas de desafiar e manipular, que visam, respectivamente, à rejeição inequívoca de normas e a expectativas institucionais; e visam propositalmente a induzir a pressões institucionais com o intuito de alterar ativamente suas expectativas.

Essas respostas estão ligadas aos fatores preditivos de multiplicidade e restrição. $O$ primeiro é relacionado aos múltiplos interessados nas organizações, como estado, sindicatos, grupos de interesse e público em geral. Quando as organizações sofrem pressões de vários stakeholders diferentes, torna-se difícil a conformação com todos eles, fazendo com que haja uma maior resistência às pressões institucionais (Oliver, 1991). O segundo está ligado à restrição imposta pelas pressões institucionais; quando estas limitam as operações das organizações é provável uma maior resistência (Oliver, 1991). 
Apesar da extensa literatura baseada na tipologia de Oliver (1991), esta tem sido pouco explorada visando ao escopo específico gerencial, como orçamento (Wijethilake et al., 2017). Um trabalho que explora as respostas estratégicas no planejamento orçamentário foi o estudo de Frezatti et al. (2007), que utilizou a tipologia de Oliver (1991), atrelada ao modelo de institucionalização de Tolbert e Zucker (1999) para investigar se existe associação entre o sucesso ao atingir as metas do planejamento tático e o tipo de resposta estratégica dos gestores em uma organização. Pode-se perceber a partir dos resultados, que os gestores, ao alcançarem metas, têm uma tendência de responder às pressões estratégicas de forma mais ativa, rejeitando as normas institucionais, confirmando o que foi proposto por Oliver. Destaca-se que os achados a partir dessa tipologia têm auxiliado na compreensão da literatura embasada pela $t E$.

\subsection{FINALIDADES DO ORÇAMENTO}

O orçamento tem sido parte integrante dos sistemas de controle gerenciais da maioria das empresas (Hansen \& Van der Stede, 2004; Kenno et al., 2018). Entretanto, apesar de sua extensa utilização, uma parte da literatura aponta para a falha nos orçamentos (Neely et al., 2001), contudo, em vez de abandonar o artefato, as organizações têm buscado alternativas para dar conta desses problemas (Libby \& Lindsay, 2010).

Ressalta-se, todavia, que a maioria dessas críticas se referem ao orçamento como ferramenta de avaliação de desempenho, enquanto as empresas utilizam os orçamentos para finalidades de planejamento e controle (Sivabalan, Booth, Malmi, \& Brown, 2009). Esses achados apontam que as características podem variar de acordo com as finalidades dadas ao orçamento.

Hansen e Van der Stede (2004) elaboram uma divisão do orçamento em quatro etapas de acordo com sua finalidade, duas delas visando ao curto prazo (i) planejamento operacional e (ii) avaliação de desempenho, e outras duas tendo em vista o longo prazo (iii) comunicação de metas e (iv) formação de estratégias. O trabalho investigou essas potenciais razões para a realização dos orçamentos nas organizações, seus antecedentes e as características que potencialmente influenciam o desempenho.

Uma alternativa, visando às finalidades do orçamento, vem do estudo de Ekholm e Wallin (2011), que testaram se os orçamentos flexíveis são melhores que os orçamentos fixos anuais. Quanto às finalidades do orçamento, os autores se basearam nas finalidades propostas por Ax \& Kullven (2005), posteriormente as dividindo em Planejamento e Diálogo, tanto para o orçamento fixo quanto para o orçamento flexível.

As finalidades propostas por Ekholm e Wallin (2011) abrangem grande parte das funções apresentadas pelos estudos anteriores. Os autores ainda agrupam as finalidades em 
Planejamento e Diálogo. Essas finalidades foram utilizadas também por Mucci et al. (2016), que analisaram a associação entre as funções de Planejamento e Diálogo e as percepções de relevância para a tomada de decisões e de utilidade para a gestão do orçamento.

A finalidade de Planejamento refere-se à elaboração de um plano a partir das estratégias organizacionais e está ligada ao planejamento, coordenação, alocação de recursos e determinação dos volumes operacionais (Ax \& Kullven, 2005; Ekholm \& Wallin, 2011; Mucci et al., 2016). A finalidade de diálogo, por sua vez, está ligada às ações tomadas após a planificação e refere-se à comunicação, criação de consciência e motivação (Ekholm \& Wallin, 2011; Mucci et al., 2016).

\subsection{HIPÓTESES DE PESQUISA}

A partir do problema de pesquisa e dos objetivos apresentados, foram levantadas as hipóteses de investigação visando refletir a influência do isomorfismo institucional na autorrejeição ao orçamento no contexto estudado, e a mediação de tal influência pelas finalidades dadas ao orçamento.

Primeiramente, espera-se que 0 isomorfismo institucional influencie positivamente a utilidade das finalidades do orçamento, visto que os resultados empíricos apontam que as pressões institucionais que levam ao isomorfismo influenciam como as organizações utilizam o orçamento e a utilização dada ao artefato.

Ugrin (2009) aponta que o isomorfismo institucional influencia a decisão de adoção de artefatos gerenciais, podendo também influenciar a sua importância, a partir da imitação de pares, conformidade com as normas e coerção de profissionais da área.

O estudo de Amans et al. (2015) ainda aponta que as pressões, maiores ou menores, podem definir como o orçamento será utilizado na organização, nesse caso, se de forma mais efetiva, tendo suas finalidades grande importância, ou cerimonial com o instrumento sendo utilizado apenas em resposta a pressões externas, sem importância para suas finalidades.

Ozdil e Hoque (2017), por sua vez, encontraram que foram realizadas mudanças no orçamento, aumentando sua importância no contexto de uma universidade pública, em resposta ao isomorfismo coercitivo e à busca por eficiência. A partir dos resultados encontrados, foi elaborada a primeira hipótese:

$\mathrm{H}_{\mathrm{i}}: \mathrm{O}$ isomorfismo institucional influencia positivamente as finalidades do orçamento

As finalidades dadas ao orçamento afetam ainda a forma como os envolvidos na organização veem o artefato (Arnold \& Gillenkirch, 2015; Kornacker, Schentler, Williams, \& 
Motwani, 2011; Mucci et al., 2016; Sivabalan et al., 2009), podendo trazer diferentes respostas, alterando a satisfação global, desempenho da unidade organizacional (Hansen \& Van der Stede, 2004; Piccoli, Warken, Lavarda, Mazzioni, \& Carpes, 2014), utilidade e relevância percebida (Mucci et al., 2016).

Visto que a utilidade das finalidades do orçamento tem relação com percepções positivas sobre o artefato (Mucci et al., 2016; Piccoli et al., 2014;), espera-se que a utilidade das finalidades apresente uma relação negativa com a autorrejeição, considerada como recusa ao artefato e/ou indução a pressões com o intuito de alterar suas expectativas. Sponem e Lambert (2016) ainda apontam que o orçamento tende a ser menos criticado quando há uma maior utilidade atribuída às suas finalidades. A partir disso, foi elaborada a segunda hipótese:

$\mathrm{H}_{2}$ : As finalidades do orçamento influenciam negativamente a autorrejeição

Oliver (1991) apresenta dois fatores preditivos que podem levar a respostas de rejeição. A primeira está ligada à multiplicidade de agentes, fazendo com que as organizações sejam confrontadas com múltiplas pressões conflitantes as quais limitam sua capacidade de conformidade. A autora complementa que exigências incompatíveis impossibilitam a conformidade, visto que a satisfação de uma pressão muitas vezes requer ignorar outras exigências (Oliver, 1991).

O outro fator apontado é a restrição, quando a norma institucional imposta, no caso o orçamento, tira a autonomia nas relações e na tomada de decisões dos envolvidos. Nesse caso, os envolvidos tendem a desafiar e manipular os níveis mais altos a fim de ter liberdade de escolha em suas operações (Oliver, 1991).

Todavia, os autores podem optar por restringir sua autonomia, em troca de uma maior legitimidade ou viabilidade econômica, em resposta às pressões institucionais que levam ao isomorfismo (Oliver, 1991). Estudos ainda trazem que a recusa é mais provável quando o isomorfismo institucional é baixo nas empresas (Amans et al., 2015; Cohen \& Karatzimas, 2014; Russo, Parisi, \& Pereira, 2016).

Em decorrência de os fatores preditivos ligados ao isomorfismo estarem mais propensos à resposta de aceitação, espera-se que o isomorfismo institucional tenha uma relação negativa com a autorrejeição. Assim, foi elaborada a terceira hipótese:

$$
\mathrm{H}_{3}: \mathrm{O} \text { isomorfismo institucional influencia negativamente a autorrejeição }
$$


Finalmente, visto o que já foi explanado quanto à influência do isomorfismo institucional nas finalidades do orçamento (Amans et al., 2015; Ozdil \& Hoque, 2017), considerando que uma maior utilização das finalidades tende a levar a menos críticas (Sponem \& Lambert, 2016) e os fatores preditivos ligados ao isomorfismo estarem mais propensos à resposta de aceitação (Oliver, 1991), espera-se que, assim como na resposta estratégica de aceitação, a relação entre isomorfismo institucional e autorrejeição seja mediada pelas finalidades do orçamento, tendo um efeito contrário na relação. Posto isso, elaborou-se a quarta hipótese:

$\mathrm{H}_{4}: \mathrm{O}$ isomorfismo institucional influencia negativamente a autorrejeição mediado pelas finalidades do orçamento

Com a definição das hipóteses a serem testadas, apresenta-se o modelo teórico do estudo:

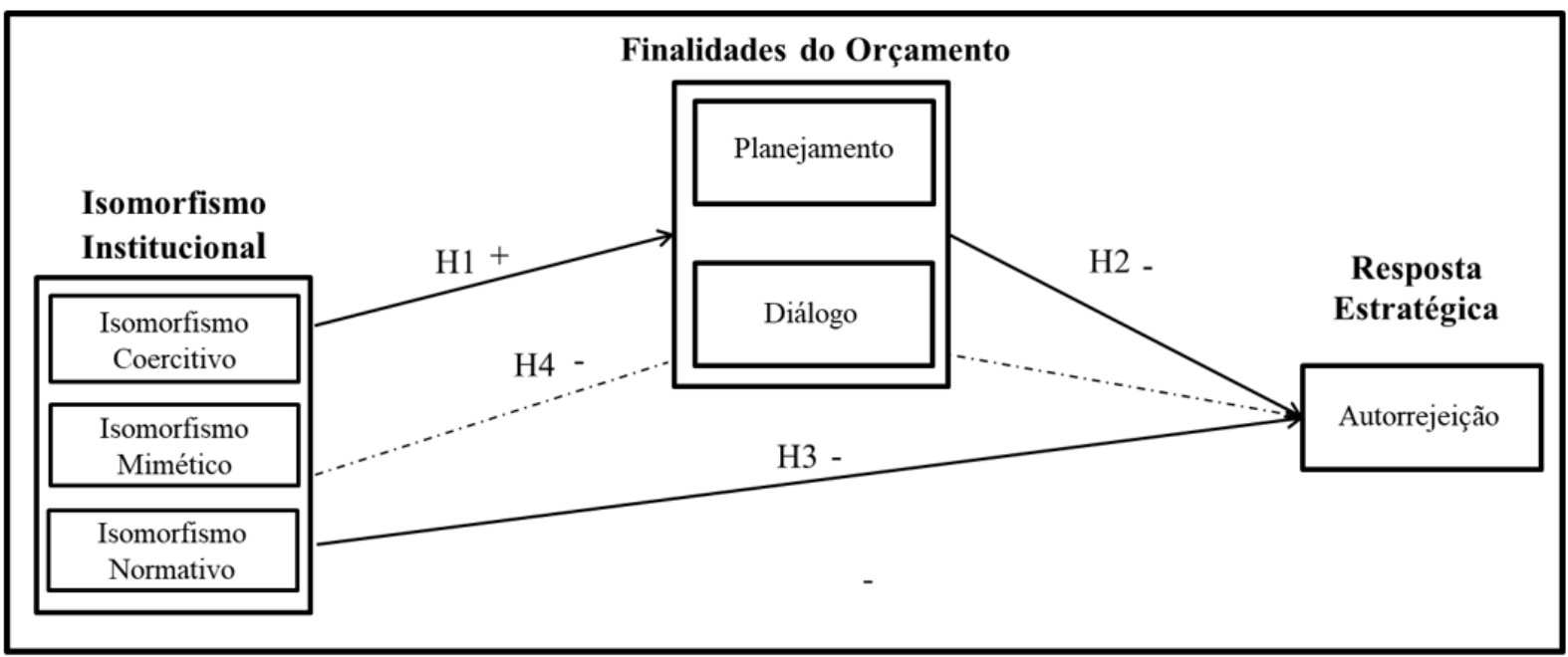

Relação Direta

Mediação

Figura 1. Modelo teórico.

Finalizadas a fundamentação teórica e a elaboração das hipóteses do estudo, na próxima seção apresenta-se a metodologia da pesquisa. 


\section{METODOLOGIA}

\subsection{CARACTERIZAÇÃO DA PESQUISA E POPULAÇÃO}

No que se refere aos objetivos, o estudo possui caráter descritivo, abordado de uma forma quantitativa e entre os procedimentos técnicos é caracterizado como pesquisa de levantamento, tipo survey (Martins \& Theóphilo, 2009).

Para o presente estudo, a população escolhida foram os gerentes do setor farmacêutico brasileiro. Buscaram-se as empresas vinculadas ao Guia da Farmácia e que tivessem sua atividade principal no setor farmacêutico, sendo encontrado um total de 121 empresas. Foram convidados a participar 4.409 gerentes das empresas selecionadas via convite de conexão na Rede Linkedin, solicitando que caso os gerentes tivessem interesse em participar, aceitassem o convite de conexão. Desses, 1.730 demonstraram interesse em participar da pesquisa, aceitando a conexão e recebendo a carta de apresentação da pesquisa e o link para o questionário. A partir disso, foram coletadas 150 respostas.

O cálculo do tamanho adequado da amostra, visando ao poder estatístico da análise, foi realizado pelo software G*Power. Para sua realização foram utilizados os critérios estabelecidos por Faul, Erdfelder, Buchner, e Lang (2009), a partir das variáveis preditoras sobre a variável independente, o tamanho do efeito médio de 0,15 , o poder da amostra de $1-\beta=0,8$ e o nível de significância de $\alpha=0,05$. A partir dos critérios foi estabelecido que para a avaliação do modelo teórico se esperava no mínimo 77 respostas. A pesquisa obteve um total de 147 respostas válidas, comprovando o tamanho adequado para as análises realizadas.

\subsection{CONSTRUCTO DE PESQUISA}

Visando cumprir o objetivo proposto, foi elaborado o constructo de pesquisa. Quanto ao isomorfismo institucional, foram utilizados os pressupostos de DiMaggio e Powell (1983). Visando à autorrejeição, foram utilizadas as respostas estratégicas de desafiar e manipular o modelo conceitual de Oliver (1991). Por fim, as finalidades do orçamento foram baseadas nos estudos de Ekholm e Wallin (2011) e Mucci et al. (2016), visando às seguintes finalidades: planejamento e diálogo. A Tabela 1 apresenta o constructo de pesquisa, com as definições das variáveis e os estudos que utilizaram a mesma definição do presente estudo: 
Tabela 1

Constructo de pesquisa

\begin{tabular}{|c|c|c|c|}
\hline Constructo & Definições & Mensuração & Estudos anteriores \\
\hline $\begin{array}{l}\text { Isomorfismo } \\
\text { Institucional }\end{array}$ & $\begin{array}{l}\text { Processo de homogeneização proveniente } \\
\text { de pressões formais e informais exercidas } \\
\text { por influências políticas de outras organi- } \\
\text { zações das quais dependem, expectativas } \\
\text { culturais, tecnologias insuficientemente } \\
\text { compreendidas, metas ambíguas ou } \\
\text { incerteza no ambiente elou decorrente da } \\
\text { profissionalização dos agentes (DiMaggio } \\
\& \text { Powell, 1983). }\end{array}$ & $\begin{array}{l}\text { Escala Likert de } 5 \text { pon- } \\
\text { tos, em que } 1 \text { representa } \\
\text { o menor grau de inten- } \\
\text { sidade e } 5 \text { o maior grau } \\
\text { de intensidade }\end{array}$ & $\begin{array}{l}\text { DiMaggio e Powell } \\
\text { (1983), Cohen e } \\
\text { Karatzimas (2014), } \\
\text { Amans et al. (2015) } \\
\text { e } \\
\text { Ozdil e Hoque } \\
\text { (2017). }\end{array}$ \\
\hline $\begin{array}{l}\text { Respostas Estra- } \\
\text { tégicas }\end{array}$ & $\begin{array}{l}\text { Rejeição inequívoca de normas e expec- } \\
\text { tativas institucionais elou alterar ativa- } \\
\text { mente ou exercer poder sobre o conteúdo } \\
\text { das próprias expectativas ou fontes que } \\
\text { buscam expressar ou aplicá-las (Oliver, } \\
\text { 1991). }\end{array}$ & $\begin{array}{l}\text { Escala Likert de } 5 \text { pon- } \\
\text { tos, em que } 1 \text { representa } \\
\text { o menor grau de con- } \\
\text { cordância e } 5 \text { a máxima } \\
\text { concordância }\end{array}$ & $\begin{array}{l}\text { Oliver (1991) e } \\
\text { Frezatti et al. } \\
\text { (2007). }\end{array}$ \\
\hline $\begin{array}{l}\text { Finalidades de } \\
\text { Planejamento }\end{array}$ & $\begin{array}{l}\text { O orçamento como artefato de planifi- } \\
\text { cação das estratégias (Ekholm \& Wallin, } \\
\text { 2011). }\end{array}$ & \multirow{2}{*}{$\begin{array}{l}\text { Escala Likert de } 5 \text { pon- } \\
\text { tos, em que } 1 \text { significa } \\
\text { Nada Útil e } 5 \text { significa } \\
\text { Muito Útil }\end{array}$} & \multirow{2}{*}{$\begin{array}{l}\text { Ekholm \& Wallin } \\
\text { (2011); Mucci et al. } \\
(2016)\end{array}$} \\
\hline $\begin{array}{l}\text { Finalidades de } \\
\text { Diálogo }\end{array}$ & $\begin{array}{l}\text { O orçamento como artefato de planifi- } \\
\text { cação das estratégias (Ekholm \& Wallin, } \\
\text { 2011). }\end{array}$ & & \\
\hline
\end{tabular}

Objetivando evitar distorções no entendimento dos respondentes, o questionário elaborado a partir do construto de pesquisa passou por pesquisadores da área para que trouxessem contribuições quanto à sua estrutura. Na sequência, profissionais que trabalham no ramo fizeram contribuições sobre a clareza das assertivas. Após as contribuições dos dois grupos quanto ao entendimento das assertivas, o questionário foi finalizado.

\subsection{COLETA E ANÁLISE DOS DADOS}

A coleta de dados foi realizada por um questionário eletrônico, com o link disponibilizado a partir do GoogleForms. A partir da população selecionada foram buscados na rede social Linkedin gerentes das mais variadas áreas das empresas listadas no Guia da Farmácia e enviado o convite informando sobre a pesquisa e seu objetivo. Quando os participantes demonstraram interesse em participar, foi enviada uma carta de apresentação da pesquisa para verificar se ele se enquadrava no perfil buscado e o link do questionário. Em alguns casos foram enviadas mais algumas breves explicações do objetivo da pesquisa aos participantes.

Foi solicitada ainda a autorização para a utilização das respostas na elaboração do presente trabalho. $O$ período de coleta de dados foi de 26 de setembro de 2018 a 16 de novembro de 2018. Estabeleceu-se contato com todos os gerentes das empresas listadas que 
possuíam conta na rede Linkedin, os quais ainda atuavam na empresa e que estavam em sedes brasileiras.

Após a coleta e tabulação dos dados, foi realizada a análise dos resultados, a partir dos seguintes procedimentos: análise descritiva, análise fatorial exploratória e modelagem de Equações Estruturais.

A análise da presente pesquisa foi realizada pelos mínimos quadrados parciais (PLSSEM), recomendados para modelos teóricos pouco explorados (Ringle, Silva, \& Bido, 2014). O software para a realização da análise também foi o SmartPLS 3. Para o teste de hipóteses e análise da significância das relações ( $p$-valor) entre as variáveis no modelo estrutural, utilizou-se o procedimento de Bootstraping (Ringle et al., 2014).

Para a mediação foram utilizados os critérios de mediação estabelecidos por Baron e Kenny (1986): a variável independente deve influenciar a mediadora; a mediadora deve influenciar a variável dependente; a variável independente deve influenciar a variável dependente; e o efeito da variável independente na variável dependente deve ser menor na mediação do que na relação direta (Baron \& Kenny, 1986). Para execução e apresentação dessa etapa, os softwares utilizados foram o Microsoft Office Excel, SPSS Statistic e SmartPLS.

\section{APRESENTAÇÃO E DISCUSSÃO DOS RESULTADOS}

\subsection{MODELO DE MENSURAÇÃO}

O primeiro passo para a utilização da modelagem de equações estruturais pelos mínimos quadrados parciais (PLS-SEM) é o teste de confiabilidade e validade do modelo de mensuração, realizado a partir dos índices de validade convergente, confiança na consistência interna e validade discriminante (Hair, Gabriel, e Patel, 2014).

As validades convergentes são obtidas por meio das observações das Variâncias Médias Extraídas (Average Variance Extracted-AVEs). A AVE analisa o quanto, em média, as variáveis se correlacionam positivamente com os seus respectivos constructos. Para esse índice, é indicado o critério de Fornell e Larcker (1981), no qual os valores das AVEs devem ser maiores que 0,5 para que o modelo apresente um resultado satisfatório (Ringle et al., 2014).

Foi observada também a consistência interna, por meio do Alfa de Cronbach (AC) e (Hair et al., 2014) pela AC é a Confiabilidade Composta (CC) (Ringle et al., 2014). Os dois índices são utilizados para observar se a amostra está livre de vieses e, se as respostas em seu conjunto são confiáveis (Hair et al., 2014; Ringle et al., 2014). 
No caso de pesquisas exploratórias, Hair et al. (2014) indicam que valores acima de 0,7 para o AC e CC são considerados adequados. A Tabela 2 apresenta a validação do modelo a partir dos critérios anteriores estabelecidos:

Tabela 2

Validação do modelo de mensuração

\begin{tabular}{lccr}
\hline & AVE & CC & AC \\
\hline Isomorfismo Institucional & 0,540 & 0,891 & 0,858 \\
\hline Planejamento & 0,633 & 0,873 & 0,805 \\
\hline Diálogo & 0,658 & 0,885 & 0,827 \\
\hline Rejeição & 0,638 & 0,873 & 0,824
\end{tabular}

Nota. AVE: Variâncias Médias Extraídas; CC: Confiabilidade Composta; AC: Alfa de Cronbach.

Para a avaliação da validade discriminante (VD), utilizada para analisar se as variáveis latentes são independentes uma das outras (Hair, 2014; Ringle et al., 2014), foi utilizado o critério de Fornell e Larcker (1981). Depois da confirmação da confiabilidade e validade do modelo estrutural, a próxima etapa foram a avaliação dos resultados do modelo estrutural a partir dos recursos preditivos do modelo e as relações entre os construtos.

\subsection{MODELO ESTRUTURAL E TESTE DE HIPÓTESES}

Após a validação do modelo de mensuração, verificou-se o $\mathrm{R}^{2}$ (Coeficiente de relação de Pearson) de cada construto, que indica o quanto cada variável dependente é explicada pelas variáveis independentes. Nos casos das pesquisas em Ciências Sociais, Cohen (1988) classifica o poder explicativo nas seguintes condições: $R^{2}=2 \%$ : efeito pequeno, $R^{2}=13 \%$ : efeito médio e $\mathrm{R}^{2}=26 \%$ efeito grande. A Tabela 3 evidencia os Coeficiente de Determinação de Pearson do modelo:

Tabela 3

Coeficientes de determinação de Pearson do modelo

\begin{tabular}{lll}
\hline Construto & $\mathrm{R}^{2}-$ Modelo sem Mediação & $\mathrm{R}^{2}$-Modelo com Mediação \\
\hline Planejamento & Não há & 0,178 \\
\hline Diálogo & Não há & 0,372 \\
\hline Rejeição & 0,068 & 0,081 \\
\hline
\end{tabular}

Percebe-se, a partir do modelo estrutural, que as variáveis latentes de finalidades do orçamento apresentam um grande poder explicativo, no caso da autorrejeição o efeito é pequeno. 
Realizadas a análise do modelo de mensuração e a avaliação do modelo estrutural ajustado, utilizou-se o procedimento de Bootstraping (Ringle et al., 2014) para análise da significância das relações ( $p$-valor) no modelo estrutural. Foram encontradas relações positivas e significantes ( $p$-valor $<0,05$ ), indicando a relação entre isomorfismo institucional, finalidades do orçamento e autorrejeição, contudo com um efeito contrário do esperado.

Tabela 4

Coeficiente de caminhos e significância das relações

\begin{tabular}{|c|c|c|c|c|c|c|c|c|c|c|}
\hline $\mathrm{H}$ & \multicolumn{3}{|l|}{ Relação } & \multicolumn{2}{|c|}{$\begin{array}{l}\text { Coeficiente estru- } \\
\text { tural }\end{array}$} & \multicolumn{2}{|c|}{ Erro padrão } & t-valor & \multicolumn{2}{|l|}{ p-valor } \\
\hline \multirow[b]{2}{*}{$\mathrm{HI}$} & \multicolumn{3}{|c|}{$\begin{array}{l}\text { Isomorfismo Institucional } \\
\text {-> Planejamento }\end{array}$} & 0,421 & & \multicolumn{2}{|l|}{0,068} & 6,189 & \multicolumn{2}{|l|}{0,000} \\
\hline & \multicolumn{3}{|c|}{$\begin{array}{l}\text { Isomorfismo Institucional } \\
\text {-> Diálogo }\end{array}$} & 0,350 & & \multicolumn{2}{|l|}{0,076} & 4,611 & \multicolumn{2}{|l|}{0,000} \\
\hline \multirow[b]{2}{*}{$\mathrm{H} 2$} & \multicolumn{3}{|c|}{ Planejamento -> Rejeição } & $-0,077$ & & \multicolumn{2}{|l|}{0,102} & 0,754 & \multicolumn{2}{|l|}{0,451} \\
\hline & \multicolumn{3}{|c|}{ Diálogo -> Rejeição } & $-0,137$ & & \multicolumn{2}{|l|}{0,120} & 1,150 & \multicolumn{2}{|l|}{0,250} \\
\hline \multirow[t]{4}{*}{$\mathrm{H} 3$} & \multicolumn{3}{|c|}{$\begin{array}{l}\text { Isomorfismo Institucional } \\
\text {-> Rejeição }\end{array}$} & 0,261 & & \multicolumn{2}{|l|}{0,088} & 2,961 & \multicolumn{2}{|l|}{0,003} \\
\hline & \multirow{3}{*}{$\begin{array}{l}\text { (C) } \\
\text { Caminhos }\end{array}$} & $\begin{array}{l}\text { Mod } \\
\text { sem } \\
\text { Med. }\end{array}$ & & \multicolumn{6}{|c|}{$\begin{array}{l}\text { Modelo com Mediação considerando Finalidades do Orçamen- } \\
\text { to }\end{array}$} & \multirow{3}{*}{$\begin{array}{l}\text { Aceite } \\
\text { da Med. }\end{array}$} \\
\hline & & Efeito & Efeito & Direto & Efeito & direto & Efeito & Total & Efeit Med. & \\
\hline & & Coef. & Coef. & $\%$ & Coef. & $\%$ & Coef. & p-valor & $\%$ & \\
\hline $\mathrm{H} 4$ & ISO> REJ & 0,261 & 0,339 & 129,88 & $-0,102$ & $-39,08$ & 0,237 & 0,000 & 9,19 & Rej. \\
\hline
\end{tabular}

Para a análise de mediação foram utilizados os critérios estabelecidos por Baron e Kenny (1986): a variável independente (isomorfismo institucional) deve influenciar significativamente a mediadora (finalidades do orçamento); a mediadora (finalidades do orçamento) deve influenciar significativamente a variável dependente (autorrejeição) e a variável independente (isomorfismo institucional) deve influenciar significativamente a variável dependente (autorrejeição).

Se os três critérios forem atendidos, para confirmar a mediação, o efeito da variável independente na variável dependente deve ser menor na mediação do que na relação direta, caracterizando a mediação parcial. Caso a variável independente não influencie de forma significativa a variável dependente após a inclusão das variáveis mediadoras, tem-se a mediação total com a variável mediadora absorvendo todo o efeito da relação (Baron \& Kenny, 1986).

A Tabela 4 apresenta as relações diretas das variáveis a partir do procedimento de Bootstraping e evidencia a avaliação da mediação a partir da comparação dos coeficientes, entre a relação direta entre isomorfismo institucional e as respostas estratégicas com os coeficientes de relação com a mediação das finalidades do orçamento. Como a autorrejeição 
não apresentou significância na relação com as finalidades do orçamento, não se pode comprovar a mediação.

\subsection{DISCUSSÃO DOS RESULTADOS}

Após a realização da modelagem de equações estruturais, a Tabela 5 apresenta a análise das hipóteses:

Tabela 5

Análise das hipóteses de pesquisa

\begin{tabular}{|c|c|c|c|}
\hline Hipótese & Relações & Sig & Decisão \\
\hline \multirow{2}{*}{$\begin{array}{l}\mathrm{H} 1: \mathrm{O} \text { isomorfismo institucional influencia positivamente as finalida- } \\
\text { des do orçamento }\end{array}$} & ISO > FPLAN & sig. & \multirow{2}{*}{ Aceita } \\
\hline & ISO > FDIA & sig. & \\
\hline \multirow{2}{*}{$\begin{array}{l}\text { H2: As finalidades do orçamento influenciam negativamente a } \\
\text { autorrejeição }\end{array}$} & FPLAN > REJ & $\mathrm{N}$ sig. & \multirow{2}{*}{ Rejeitada } \\
\hline & FDIA > REJ & $\mathrm{N}$ sig. & \\
\hline $\begin{array}{l}\mathrm{H} 3: \mathrm{O} \text { isomorfismo institucional influencia negativamente a autorre- } \\
\text { jeição }\end{array}$ & ISO > REJ & sig. & Rejeitada \\
\hline $\begin{array}{l}\text { H4: O isomorfismo institucional influencia negativamente a autore- } \\
\text { jeição mediada pelas finalidades do orçamento }\end{array}$ & $\begin{array}{l}\text { ISO > FINOR } \\
>\text { REJ }\end{array}$ & sig. & Rejeitada \\
\hline
\end{tabular}

Nota. ISO: Isomorfismo Institucional; FPLAN: Finalidade de Planejamento; FDIA: Finalidade de Diálogo; ACE: Aceitação do orçamento; REJ: Rejeição do Orçamento; FINOR: Finalidades do Orçamento.

A Hipótese 1 foi aceita, visto que houve relação positiva e significativa do isomorfismo institucional com as finalidades do orçamento de Planejamento e Diálogo. O achado confirma estudos anteriores, tendo o orçamento uma maior utilidade em resposta ao isomorfismo institucional (Amans et al., 2015; Ozdil \& Hoque, 2017).

Conforme já destacado, o setor estudado tende a sofrer do isomorfismo institucional coercitivo, mimético e normativo, visto que as empresas enfrentam regulações econômicas, controle de preços pela Câmara de Regulação do Mercado de Medicamentos (CMED) e um crescente desenvolvimento econômico que tende a levar a maiores incertezas e à profissionalização dos envolvidos. Com parcimônia, a partir dos números apresentados, acredita-se que, em resposta ao isomorfismo institucional, os gerentes têm dado uma maior utilidade às finalidades do orçamento.

A Hipótese 2, no entanto, foi rejeitada em razão de as relações entre as finalidades de planejamento e diálogo com a autorrejeição não apresentarem significância, apesar de serem negativas como o esperado. Os resultados indicam que as finalidades não têm influência significativa estatisticamente na minimização da autorrejeição. Com isso, devem ser incluídas novas variáveis para a investigação do que pode auxiliar na redução à rejeição 
ao artefato, o que consequentemente poderia aumentar a aceitação dos envolvidos, sua satisfação global e o desempenho organizacional.

Salienta-se que houve um coeficiente negativo maior entre as finalidades de diálogo e a autorrejeição em comparação com as finalidades de planejamento. Uma possível explicação é que as finalidades visam à comunicação, ou seja, criação de consciência e motivação, o que tende a minimizar as pressões contra e/ou a recusa ao artefato.

A Hipótese 3 também foi rejeitada, uma vez que a relação entre isomorfismo institucional e autorrejeição teve uma relação positiva e significativa ao contrário do esperado. $O$ achado vai de encontro à literatura de Oliver (1991), visto que os fatores que levam às respostas estratégicas ativas apontadas pela autora são a multiplicidade, quando o estado, profissões e grupos de interesse impõem uma variedade de leis, regulamentos e expectativas sobre a organização e a restrição, quando há perda na liberdade organizacional.

A partir dos resultados encontrados, presume-se que os gerentes veem uma multiplicidade de interesses no orçamento das empresas, levando a uma maior probabilidade de respostas de desafio ou manipulação. Essa multiplicidade pode ser explicada pela diversidade de cargos de funções dos gerentes estudados. Mucci et al. (2016) apontam que cada área pode apresentar uma visão diferente ao orçamento, que pode gerar essa multiplicidade.

Outra possibilidade é que o orçamento tem restringido suas atividades, fazendo com que os gerentes, com a diretoria, optem por recusar o artefato, tentando mudar os objetivos propostos e as demandas relacionadas ao orçamento, visto que as assertivas ligadas a contestar o orçamento foram as que tiveram as maiores médias. Oliver (1991) aponta que a ameaça à autonomia é mais suscetível a estratégias de manipulação, como foi encontrado no contexto estudado. Uma possível explicação nesse ponto seria que o estudo envolveu gerentes, que já recebem parte ou todo o orçamento dos setores estratégicos, e a partir disso estes acreditam que o artefato tem restringido sua autonomia.

Por fim, a Hipótese 4 foi rejeitada, visto que não foi atendido um dos critérios estabelecidos por Baron e Kenny (1986) de a variável mediadora (finalidades do orçamento) ter influência significante estatisticamente sobre a variável dependente (autorrejeição), mesmo com os outros quesitos sendo atendidos.

Destaca-se que a relação direta entre isomorfismo institucional e autorrejeição mediada pelas finalidades do orçamento apresentou um coeficiente mais baixo em comparação com a relação direta das variáveis, porém, como a Hipótese 3, a relação encontrada foi contrária ao esperado, tendo o isomorfismo institucional uma relação positiva com a autorrejeição.

Os achados sugerem que as mesmas pressões que levam a uma maior utilidade das finalidades influenciam a autorrejeição. Além das possíveis explicações apresentadas na Hipótese 3, multiplicidade e restrições, peculiaridades do setor podem ser possíveis fatores 
que levam à rejeição, sendo o artefato mais utilizado pela regulação econômica imposta do que pela busca por sustentabilidade financeira e organizacional. Tal fato abre uma oportunidade que pode ser explorada em futuros trabalhos, visto que estudos do artefato no contexto estudado ainda são escassos, como aponta Gomes et al. (2014).

\section{CONSIDERAÇÕES FINAIS}

A presente pesquisa teve como objetivo examinar a influência do isomorfismo institucional na autorrejeição, mediada pelas finalidades do orçamento. Diante disso, foram identificadas as empresas do setor farmacêutico vinculadas ao Guia da Farmácia, tendo uma amostra de 147 gerentes de 121 empresas do ramo.

A confirmação da Hipótese 1 demonstrou que o orçamento é utilizado em resposta aos mecanismos isomórficos e que $O$ isomorfismo institucional influencia positivamente as finalidades do artefato. Sugere-se também, a partir da Hipótese 2, que a maior utilização das finalidades do orçamento não influencia a rejeição ao artefato, apesar do coeficiente negativo encontrado. Todavia, foi encontrada também uma associação positiva e significativa entre o isomorfismo institucional e a autorrejeição ao orçamento, na Hipótese 3.

O achado aponta que, além das proposições apontadas, as razões para a utilização do orçamento podem estar ligadas à multiplicidade de agentes e principalmente a fatores restritivos, que podem ser relacionados ao alto isomorfismo coercitivo no setor, fazendo que o orçamento tenha, além de um caráter de legitimação e dependência, um posto de restrição na tomada de decisões dos gerentes.

Tem-se ainda, a partir das assertivas propostas, que essa rejeição não faz com que os envolvidos fujam do artefato ou utilizem um orçamento próprio, a estratégia utilizada é de pressionar a manutenção com a diretoria e tentar mudar as demandas propostas. Com isso, aponta-se que a resposta estratégica engloba a rejeição ao artefato e/ou a forma como ele é instituído na empresa, tendo no contexto uma maior incidência da segunda opção, o que corrobora a ideia de que a autorrejeição é motivada pelo fator restritivo. Entretanto, destaca-se o alto desvio nas respostas de rejeição, a partir das quais se conclui que essa rejeição não é vista em todo o contexto estudado, mas apenas em casos específicos.

Com isso, infere-se que existem focos de rejeições ao orçamento entre os gerentes do setor, porém essa rejeição visa mais à adequação de objetivos propostos do que ao abandono do instrumento. Em termos práticos, os gestores do setor farmacêutico poderiam buscar, com os gerentes operacionais, os fatores que acarretam maior autorrejeição, como a citada multiplicidade de interesses e restrição com vistas a uma melhor adequação do artefato aos níveis operacionais, e uma maior utilidade das finalidades de planejamento e diálogo no contexto estudado. 
Outra contribuição teórica é a utilização do modelo conceitual de Oliver (1991), no contexto orçamentário, visto que os estudos a partir dessa tipologia no contexto gerencial ainda são incipientes, como apontam Wijethilake et al. (2017), não tendo sido encontrados resultados no setor farmacêutico, que nacionalmente se destaca por seus expressivos números positivos e as peculiaridades coercitivas apresentadas pelo setor.

Posto que a Hipótese 3 teve um coeficiente de caminho contrário ao expectado, referente à relação do isomorfismo com a autorrejeição, futuros estudos podem, a partir da tipologia de Oliver (1991), buscar uma melhor compreensão de quais são os fatores do isomorfismo institucional que levam à autorrejeição do artefato gerencial, explorando, ainda, como os fatores preditivos apontados pela autora como propensos à rejeição, multiplicidade e restrição, relacionam-se com os mecanismos do isomorfismo institucional.

Estudos adicionais poderiam ainda utilizar, assim como o modelo conceitual de Oliver (1991), uma diferenciação entre desafiar e manipular, explorando quais são os fatores que levam os envolvidos a rejeitar o orçamento e quais levam a tentar modificar suas demandas. Tais caminhos poderiam levar a uma melhor compreensão do isomorfismo pelos envolvidos no orçamento em nível dos gerentes. Por fim, sugere-se também a aplicação do modelo proposto em outros setores com diferentes peculiaridades, para uma melhor compreensão dos achados e fortalecimento da Teoria.

\section{REFERÊNCIAS}

Amans, P., Mazars-Chapelon, A., \& Villesèque-Dubus, F. (2015). Budgeting in institutional complexity: The case of performing arts organizations. Management Accounting Research, 27, 47-66.

Arnold, M. C., \& Gillenkirch, R. M. (2015). Using negotiated budgets for planning and performance evaluation: An experimental study. Accounting, organizations and society, 43, $1-16$.

Ax, C., \& Kullven, H. (2005). Den nya Ekonomistyrningen. Upplaga 3. (3rd ed.). Malmo, Sweden: Liber.

Baron, R. M., \& Kenny, D. A. (1986). The moderator-mediator variable distinction in social psychological research: Conceptual, strategic, and statistical considerations. Journal of personality and social psychology, 51(6), 1173.

Brandau, M., Endenich, C., Trapp, R., \& Hoffjan, A. (2013). Institutional drivers of conformity-Evidence for management accounting from Brazil and Germany. International Business Review, 22(2), 466-479. 
Cohen, J. (1988). Statistical power analysis for the behavioral sciences. (2nd ed.). New York, USA: Psychology Press.

Cohen, S., \& Karatzimas, S. (2014). Reporting performance information in the public sector: The moral behind the (non) application of program budgeting in Greece. International Review of Administrative Sciences, 80(3), 619-636.

Covaleski, M., Evans III, J. H., Luft, J., \& Shields, M. D. (2003). Budgeting research: Three theoretical perspectives and criteria for selective integration. Handbooks of management accounting research, 2, 587-624.

DiMaggio, P., \& Powell, W. W. (1983). The iron cage revisited: Collective rationality and institutional isomorphism in organizational fields. American sociological review, 48(2), 147 160.

Ekholm, B. G., \& Wallin, J. (2011). The impact of uncertainty and strategy on the perceived usefulness of fixed and flexible budgets. Journal of Business Finance \& Accounting, 38(1 2), 145-164.

Faul, F., Erdfelder, E., Buchner, A., \& Lang, A. G. (2009). Statistical power analyses using G* Power 3.1: Tests for correlation and regression analyses. Behavior research methods, 41(4), 1149-1160.

Fornell, C., \& Larcker, D. F. (1981). Evaluating structural equation models with unobservable variables and measurement error. Journal of marketing research, 18(1), 39-50.

Frezatti, F., Braga de Aguiar, A., \& José Rezende, A. (2007). Strategic responses to institutional pressures, and success in achieving budget targets: A survey at a multinational company. International Journal of Accounting \& Information Management, 15(2), 50-66.

Gomes, R. D. P., Pimentel, V. P., Cardoso, M. L., \& Pieroni, J. P. (2014). O novo cenário de concorrência na indústria farmacêutica brasileira. BNDES Setorial, 39, 97-134.

Guerreiro, R., Pereira, C. A., Rezende, A. J., \& Aguiar, A. B. D. (2010). Fatores determinantes do processo de institucionalização de uma mudança na programação orçamentária: Uma pesquisa-ação em uma organização brasileira. Revista de contabilidade do mestrado em ciências contábeis da UERJ, 10(1), 59-76.

Hair, J. F., Jr., Gabriel, M. L. D. D. S., \& Patel, V. K. (2014). Modelagem de Equações Estruturais Baseada em Covariância (CB-SEM) com o AMOS: Orientações sobre a sua aplicação como uma Ferramenta de Pesquisa de Marketing. Revista Brasileira de Marketing, 13(2), 44-55. 
Hansen, S. C., \& Van der Stede, W. A. (2004). Multiple facets of budgeting: An exploratory analysis. Management accounting research, 15(4), 415-439.

Hansen, S. C., Otley, D. T., \& Van der Stede, W. A. (2003). Practice developments in budgeting: An overview and research perspective. Journal of management accounting research, 15(1), 95-116.

Kenno, S. A., Lau, M. C., \& Sainty, B. J. (2018). In search of a theory of budgeting: A literature review. Accounting perspectives, 17(4), 507-553.

Kornacker, J., Schentler, P., Williams, H. J., \& Motwani, J. (2011). Critical success factors for budgeting systems in the German context: An empirical analysis. International Journal of Business Excellence, 4(6), 621-644.

Libby, T., \& Lindsay, R. M. (2010). Beyond budgeting or budgeting reconsidered? A survey of North-American budgeting practice. Management accounting research, 21(1), 56-75.

Martins, G. D. A., \& Theóphilo, C. R. (2009). Metodologia da investigação científica. São Paulo: Atlas.

Meyer, J. W., \& Rowan, B. (1977). Institutionalized organizations: Formal structure as myth and ceremony. American journal of sociology, 83(2), 340-363.

Mucci, D. M., Frezatti, F., \& Dieng, M. (2016). As múltiplas funções do orçamento empresarial. RAC-Revista de Administração Contemporânea, 20(3).

Neely, A., Sutcliff, M. R., \& Heyns, H. R. (2001). Driving value through strategic planning and budgeting. New York: Accenture.

Oliver, C. (1991). Strategic responses to institutional processes. Academy of management review, 16(1), 145-179.

Ozdil, E., \& Hoque, Z. (2017). Budgetary change at a university: A narrative inquiry. The British Accounting Review, 49(3), 316-328.

Piccoli, M. R., Warken, I. L. M., Lavarda, C. E. F., Mazzioni, S., \& Carpes, A. da S. (2014). Os múltiplos usos do orçamento em empresas catarinenses. Unoesc \& Ciência-ACSA, 5(2), 195-206.

PricewaterhouseCoopers (2018). O Setor farmacêutico no Brasil. São Paulo.

Ringle, C. M., Silva, D. da, \& Bido, D. D. S. (2014). Modelagem de equações estruturais com utilização do SmartPLS. Revista Brasileira de Marketing, 13(2), 56-73. 
Russo, P. T., Parisi, C., \& Pereira, C. A. (2016). Evidências das forças causais críticas dos processos de institucionalização e desinstitucionalização em artefatos da contabilidade gerencial. Revista Contemporânea de Contabilidade, 13(30), 2.

Scapens, R. W. (2006). Understanding management accounting practices: A personal journey. The British Accounting Review, 38(1), 1-30.

Sindusfarma. (2018). Perfil da indústria e aspectos relevantes do setor. São Paulo.

Sivabalan, P., Booth, P., Malmi, T., \& Brown, D. A. (2009). An exploratory study of operational reasons to budget. Accounting \& Finance, 49(4), 849-871.

Sponem, S., \& Lambert, C. (2016). Exploring differences in budget characteristics, roles and satisfaction: A configurational approach. Management Accounting Research, 30, 47-61.

Tolbert, P. S., \& Zucker, L. G. (1999). The institutionalization of institutional theory. In S. R. Clegg, \& C. Hardy (Eds.), Studying Organization. Theory \& Method (pp. 169-184). London, Thousand Oaks, CA: Sage.

Ugrin, J. C. (2009). The effect of system characteristics, stage of adoption, and experience on institutional explanations for ERP systems choice. Accounting Horizons, 23(4), 365 389.

Wijethilake, C., Munir, R., \& Appuhami, R. (2017). Strategic responses to institutional pressures for sustainability: The role of management control systems. Accounting, Auditing \& Accountability Journal, 30(8), 1677-1710.

Zucker, L. G. (1987). Institutional theories of organization. Annual review of sociology, 13(1), 443-464.

\section{Como citar este artigo:}

\section{ABNT}

LUZ, Igor Pereira da; LAVARDA, Carlos Eduardo Facín. As finalidades do orçamento na influência do isomorfismo institucional na autorrejeição. RACE, Revista de Administração, Contabilidade e Economia, Joaçaba: Editora Unoesc, v. 19, n. 1, p. 54-74, jan./abr. 2020. Disponível em: http://editora.unoesc.edu.br/index.php/race. Acesso em: dia/mês/ano.

\section{APA}

Luz, I. P. da; \& Lavarda, C. E. F. (2020). As finalidades do orçamento na influência do isomorfismo institucional na autorrejeição. RACE, Revista de Administração, Contabilidade e Economia, 19(1), 54-74. Recuperado de http://editora.unoesc.edu.br/index.php/race 